\title{
SUITABILITY OF BI-VACANCY TRAP FOR TL EMISSION IN KCI
}

\author{
Ruchi Pandey ${ }^{1 a}$, S.D. Pandey ${ }^{2 a^{*}}$, Sumit Kumar ${ }^{3 b}$ and Rajendra Kumar ${ }^{4 c}$
}

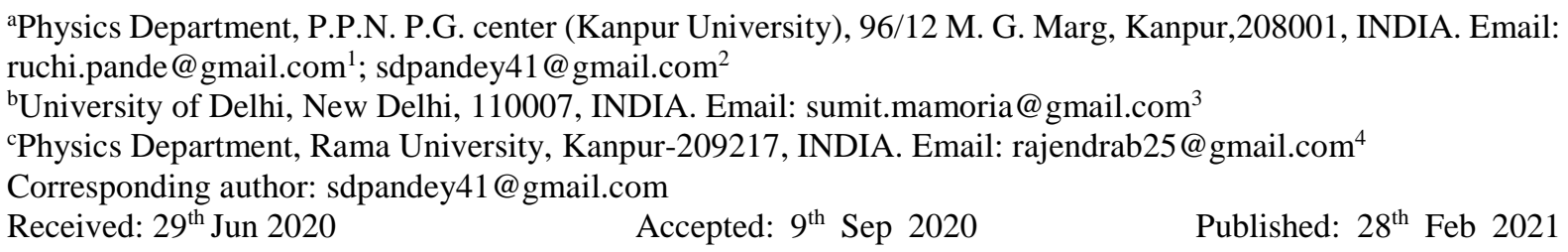

DOI: https://doi.org/10.22452/mjs.vol40no1.4

\begin{abstract}
In $\mathrm{KCl}$ crystals, the $2 \mathrm{~s}$ energy level of F-center within an anion-cation vacancy pair has been proposed as a suitable trap for thermoluminescence (TL) emission. It is further seen that the $2 \mathrm{~s}$ - level of F-center situated in cation - anion vacancy pair has large trap depth. The observed trap depth of F-center is explainable on the basis of Herman and Barnett model, modified presently and supports bi-vacancy configuration for traps in $\mathrm{KCl}$ lattice.
\end{abstract}

Keywords: TL, Traps, Trap depth, I-V pair, KCl

\section{INTRODUCTION}

As far as thermoluminescence (TL) emission is concerned, the electrons traps are supposed to get excited to the conduction band on external heating and then fall into the hole traps or recombination centers (Srivastava, 2008; Furetta, 2003; Bos, 2006).The temperature, however remains responsible for lifting of electrons from the trap to the conduction band. These traps have an important role in the TL process. The electrons situated in traps do not lose energy to drop to the lower available states. There have been numerous studies which characterize the electron traps, and their applications in dosimetry, but the nature and the physical structure of such traps in alkali halides are not yet fully established.

TL studies in a number of alkali halides have been reported (Deshmukh \& Moharil, 1985). Their studies do not, however, yield any in depth information about the traps involved. Other workers (Jain \& Mahendru, 1965) have reported TL peaks at $135{ }^{\circ} \mathrm{C}$ and $190{ }^{\circ} \mathrm{C}$ in melt grown pure $\mathrm{KCl}$ crystals. They further propose that the two peaks are due to electrons trapped at two different kinds of F-centers with trap depths of $1.05 \mathrm{eV}$ and $1.15 \mathrm{eV}$ respectively. The first type of F-centers are due to vacancies present in the crystal prior to X-ray irradiation and the second type to further vacancies generated during X-ray irradiation, perhaps near a defect. The results of above workers (Jain and Mahendru, 1965) further indicate that added calcium impurity of $50 \mathrm{ppm}$ in $\mathrm{KCl}$, introduces a new TL peak at $85^{\circ} \mathrm{C}$, enhances $135^{\circ} \mathrm{C}$ and suppresses the peak at $190^{\circ} \mathrm{C}$.

The TL studies in gamma irradiated Harshaw $\mathrm{KCl}$ crystals are reported (Ausin \& Rivas, 1972) and the role of F-centers as recombination centers in the annealing process is proposed. Other authors (Ranita et al., 2006) have 
devoted their study to explore the spectroscopy of traps in powdered $\mathrm{KCl}$ and found that trap depth is proportional to glow peak temperature $\mathrm{T}_{\mathrm{m}}$. Furthermore, a complex energy distribution of traps has been obtained (Randall \& Wilkins, 1945) in the range $0.2 \mathrm{eV}$ to $1.0 \mathrm{eV}$ in alkaline earth sulphides. These results, however, cannot give any clue to traps in $\mathrm{KCl}$.

In a previous work (Pandey et al., 2017) two TL peaks were observed at $361 \mathrm{~K}$ and $413 \mathrm{~K}$ in aqueous solution grown undoped $\mathrm{KCl}$ crystals (Sample-1) and only one TL peak at $339 \mathrm{~K}$ in $\mathrm{KCl}$ crystals grown with added $1 \% \mathrm{MnCl}_{2}$ in mother solution (Sample-2). The peak at $413 \mathrm{~K}$ in Sample-1 has been attributed to electrons liberated from anion vacancycation vacancy pair (bi-vacancy) traps which are not affected by the stray $(0.001 \%)$ impurities and the peak at $361 \mathrm{~K}$ on the other hand to electrons liberated from traps affected by stray impurities. These workers had concluded the peak at $413 \mathrm{~K}$ to be due to F-centers in bi-vacancy due to the fact that it was completely eliminated in samples with heavy dose of divalent cations. The trap depths were calculated using a standard formula (Chen, 1969) using the width of the glow peak on the fall off side. The trap depths were found to be $0.90 \mathrm{eV}$ for the peak at $413 \mathrm{~K}$ and $0.44 \mathrm{eV}$ for the peak at $361 \mathrm{~K}$. The trap depth of the only peak in $\mathrm{Mn}^{2+}$ doped $\mathrm{KCl}$ was further found to be 0.48 eV. Again, TL studies in $\mathrm{KBr}$ crystals have been made (Laad, 2008) and trap depth of $0.93 \mathrm{eV}$ is obtained.

The trap depths in $\mathrm{KCl} / \mathrm{KBr}$ hosts as obtained by different workers are given in Table-1. However, it seems that no attempt has been made so far to understand how in $\mathrm{KCl}$ crystal traps are created and what is their structure.
Our work herewith is towards establishing a model trap configuration in $\mathrm{KCl}$ for $\mathrm{TL}$ emission and explaining the trap depths obtained experimentally.

\section{THE TRAP MODEL PROPOSED}

The existence of a trap center is a crucial requirement of the TL process. An electron occupying a secluded anion vacancy is said to form a normal F-center (Figure 1a). An electron trapped in such a center would spontaneously move down the ladder of energy levels emitting photons and accompanying phonons. Thus a secluded anion vacancy cannot be a suitable trap for the TL process. We propose that an anion-cation vacancy pair termed as bivacancy (Figure 1b) can however be a suitable trap. It is known that the selection rules of dipole transitions for the case of an electron trapped in a spherical potential well are affected by the symmetry of the potential well (Davydov, 1965). In our bivacancy model of an electron trap, the extra cation vacancy coupled with the anion vacancy distorts the spherical potential well, and hence the symmetry is broken. If the potential well is not spherically symmetric, there would be new selection rules which weaken (or forbid) the previously allowed transitions. This prevents the electron in the trap to continually fall into lower energy levels.

A bi-vacancy is therefore a good candidate configuration for the TL process in an alkali halide crystal. As will be mentioned later in the text, F-centers in vacancy pairs further affected by impurity - vacancy (I-V) pairs also act as traps for electrons under different situations and lead to TL at different temperatures ( Figure 1c and 1d ). 
The traps are supposed to be situated at dislocation sites in a $\mathrm{KCl}$ crystal lattice. The two works (Benia et al., 2010 \& Wosinski, 1989) point in this direction. Though a bi-vacancy is a neutral entity, this would distort the lattice arrangement in the surrounding and lead to dislocation like situation. An electron trapped at the anion vacancy end of bi-vacancy would represent an F-center simultaneously coupled to a cation vacancy. The energy of 2 s level in such an F-center will be modified and perhaps lowered.

In a divalent cation doped $\mathrm{KCl}$, there would be plentiful I-V (ImpurityVacancy) pairs as depicted in Fig (1c) and (1d). Now, if a doped $\mathrm{KCl}$ crystal is exposed to ionizing radiations to create $\mathrm{F}$ centers, some of these would also be under the influence of the electric field of I-V pairs (Bushnell, 1964).

Considering the case of F-center in a modified arrangement (Figure1c and 1d) with the effect of I-V pairs, it is clear that $2 s$ energy level becomes shallower with values $-0.48 \mathrm{eV}$ and $-0.44 \mathrm{eV}$ depending on the nature of different doped divalent impurities. Thus, our model of trap center in a vacancy-pair as affected by I-V pairs points to the observed variation in trap depths and supports the presented ideal trap configuration in $\mathrm{KCl}$.

If we compare the trap depths for Fcenters in $\mathrm{KCl}$ as per different workers, we find that glow peak at $413 \mathrm{~K}$ in one of the works (Pandey et al., 2017) is close to the glow peak at 408K of the other group (Jain \& Mahendru, 1965) and the corresponding trap depths are quite close to each other. The latter group observed a peak at $463 \mathrm{~K}$ as well. Its presence is probably due to the fact that their $\mathrm{KCl}$ crystals were grown through melt and therefore likely to include extra defects which lead to the creation of second stage F-centers with a trap depth of $1.15 \mathrm{eV}$. Also, they did not observe the glow peak at $361 \mathrm{~K}$ seen by the first group, (Pandey et al., 2017) as the $\mathrm{KCl}$ samples of latter workers had very little divalent impurities.

\subsection{Energy lowering of F-center 2s- level in bi-vacancy}

The properties of the electron trapped inside a vacancy, can be explained by the quantum mechanical description of a particle in a spherical cavity. We choose to work with the 3-dimensional Schrödinger's equation in spherical polar coordinates, which is written as,

$$
-\frac{\hbar^{2}}{2 m}\left[\frac{1}{r^{2}} \frac{\partial}{\partial r}\left(r^{2} \frac{\partial}{\partial r}\right)+\frac{1}{r^{2} \sin \theta} \frac{\partial}{\partial \theta}\left(\sin \theta \frac{\partial}{\partial \theta}\right)+\frac{1}{r^{2} \sin ^{2} \theta} \frac{\partial^{2}}{\partial \phi^{2}}\right] \psi+V(r) \phi=E \psi
$$

Where $\mathrm{V}(\mathrm{r})=-\mathrm{V}_{0}$ for $\mathrm{r} \leq \mathrm{a}$ and $\mathrm{V}(\mathrm{r})=0$ otherwise. The wave function can now be written in a separable form as

$$
\psi(r, \theta, \phi)=R(r) Y(\theta, \phi)
$$

With,

$$
\chi(r)=R(r) / r .
$$


To calculate energies, we need only radial part of the equation, which is written as

$$
\begin{aligned}
& -\frac{\hbar^{2}}{2 m} \frac{d^{2} \chi}{d r^{2}}-V_{0} \chi \quad=E \chi \quad(r \leq a) \\
& -\frac{\hbar^{2}}{2 m} \frac{d^{2} \chi}{d r^{2}} \quad=E \chi \quad(r>a)
\end{aligned}
$$

Thus, the radial motion is equivalent to the one dimensional motion of a particle in the potential, and is given by

$$
V(r)=-V_{0}+\frac{l(l+1) \hbar^{2}}{2 m r^{2}}
$$

If we are interested only in the energy eigenvalues of the $s$-states, i.e. $l=0$, the potential becomes,

$$
V(r)=-V_{0}
$$

Solutions of equations (2) and (3) under the boundary conditions that $R$ becomes finite as $r \rightarrow 0$ and $R$ vanishes as $r \rightarrow \infty$, take the following form

$$
\begin{gathered}
\chi=A \sin (\alpha r) \quad(r \leq r) \\
\chi=A \sin (-\beta r) \quad(r>r)
\end{gathered}
$$

Where

$$
\begin{aligned}
& \alpha=\sqrt{\frac{2 m}{\hbar^{2}}\left(V_{0}-|E|\right)} \\
& \beta=\sqrt{\frac{2 m}{\hbar^{2}}|E|}
\end{aligned}
$$

The two equations (6) and (7) can now be solved graphically or numerically. We can put $\xi=\alpha a$ and $\eta=\beta a$, to obtain the following set of equations,

$$
\begin{aligned}
\xi \cot \xi & =-\eta \\
\xi^{2}+\eta^{2} & =\frac{2 m}{\hbar^{2}} V_{0} a^{2}
\end{aligned}
$$

Thus the $s$-state energy level may be obtained graphically by the intersection of equations (10) and (11) in the first quadrant, or simultaneously solving them numerically.

If we know the values of $a$ and $\mathrm{E}_{\mathrm{ns}}$, the energy of the electron in the ns state, one can find the value of $V_{0}$ and the number and values of energies of the bound electron in the well.
The arrangement of ions around electrons occupying an anion vacancy is shown in Figure 2a while for electrons at an anion vacancy end of bi -vacancy it is as shown Figure 2b. In Figure 2a, the neighborhood of electrons has octahedral symmetry. This was approximated as a spherical potential well (Herman and Barnett, 1982). For an electron in bivacancy there is however much more space to move about in the distorted spherical well. 
The results of various calculations for well depth and 2 s energy levels for the two types of wells are as follows:

\subsubsection{Spherical well for electron at anion vacancy}

The earlier workers (Herman and Barnett, 1982) presumed the width of the well to be the sum of nearest neighbor separation and the radius of the cation. These workers however, obtained the energy of 2 s level of F-center to be as low as $-2.04 \mathrm{eV}$, which is quite far off the experimental value and is not acceptable. To solve this problem we have taken the width of the well as the sum of nearest neighbor separation and the radius of the cation. With this assumption, the spherical well covers almost all available open space between the neighboring ions, but avoids including non-penetrable space of the anions. The estimated well width is thus 7.202 a.u.

The energy of $2 \mathrm{~s}$ level in $\mathrm{KCl}$ was found close to the conduction level and nearly zero (Gourary \& Adrian, 1957). For this value of $2 \mathrm{~s}$ energy level, the $\mathrm{V}_{0}$ for the spherical well comes out to be $6.64 \mathrm{eV}$. Therefore, the estimated values of the parameters in our model leads to results which are much closer to the experimental results, in contrast to the previous model (Herman and Barnett, 1982).

\subsubsection{Distorted spherical well for electron in bi-vacancy}

In this case, due to the existence of the bi-vacancy, the effective size of the spherical well is slightly increased. In this case, if we evaluate the size of the well numerically, the radii in all six mutually perpendicular directions do not turn out to be equal. In five directions, the radius is $3.81 \mathrm{~A}^{\mathrm{o}}$, just like in the case of the perfect spherical well, while the radius along the sixth direction is $6.27 \mathrm{~A}^{\mathrm{o}}$, the well surface passing through the center of the outer anion vacancy. This distortion in the sphere also takes care of the open space between the two anion pairs as shown in the Figure $2 \mathrm{~b}$. The estimated value of the width now becomes, $\mathrm{a}=4.22 \mathrm{~A}^{\mathrm{o}}=7.962$ a.u. The experimental value obtained (Pandey et al., 2017) for the $E_{2 s}$ is $-0.9 \mathrm{eV}$, and using this, we found the depth of the potential well to be $\mathrm{V}_{0}=6.15 \mathrm{eV}$.

It can be seen that the depth of required potential well decreases for bivacancy with respect to the simple anion vacancy trap. This result supports the view of classical electrodynamics that out of six nearest neighbor charges of anion vacancy one is eliminated in the bi-vacancy environment.

In our present bi-vacancy model, we approximated the distorted potential well by a spherical well of certain width and depth. A better approach would however be to solve 3D-Schrodinger wave equation for a more realistic spheroidal potential well and this may be done in our future work.

\subsection{Raising of level through electric field of I-V pairs}

Considering the case of F-center in modified arrangements (Figure 1c and 1d) with the influence of I-V pairs, we find that 2s level at $0.9 \mathrm{eV}$ becomes shallower (Pandey et al., 2017) with energy depths of about $0.48 \mathrm{eV}$ and $0.44 \mathrm{eV}$, depending on the nature of different doped divalent impurities.

It is known through a recent work (Pandey et al., 2020) that divalent cations substituting in alkali halide crystals lead to the creation of associated cation vacancies necessary for local charge compensation. 
The I-V pairs so created are associated with $\mathrm{F}$-centers as in Figs. 1c and 1d in nnn and $\mathrm{nn}$ arrangements. The electric field of such dipoles affects the various energy levels of F-center in the new arrangement. The $2 \mathrm{~s}$ levels in the well are thus raised in energy in this process due to the Stark effect of I-V pairs' electric field. The process occurs as per second order perturbation theory, the effect being proportional to the square of the electric field. The energy of $2 \mathrm{~s}$ level which was $0.9 \mathrm{eV}$ is thus elevated to 0.44 $\mathrm{eV} / 0.48 \mathrm{eV}$ as per the nature of stray or doped impurities. The theory developed by the above workers (Pandey et al., 2020) thus indirectly supports the existence of deep traps in undoped $\mathrm{KCl}$ crystals.

\section{MORE ABOUT BI-VACANCY TRAPS}

\subsection{Effect of temperature on trap model}

The binding energy of bi-vacancy in $\mathrm{NaCl}$ is about $1.0 \mathrm{eV}$ (Tharmalingam \& Lidiard, 1961). Thus, at temperatures much below the melting point of alkali halide host, with average thermal energy $(k T)$ of about $0.05 \mathrm{eV}$, the modes of crystal are not likely to transfer enough energy to the surroundings of bi-vacancy to cause its dissociation.

Similarly, lattice modes are not likely to cause a change in trap depth values with temperature and will not affect the TL emission in any way. This trap model would be valid as long as bi-vacancy does not break.

\subsection{Elimination of bi-vacancies due to doping}

The doping of an alkali halide crystal with divalent cations, would cause a reduction in its free cation concentration and thus lead to reduction of bi-vacancy formation as well; in this process $\mathrm{Cl}^{-}$ions would be created. The suppression of TL peaks in $\mathrm{KCl}$ due to doping of $\mathrm{Mn}^{2+}$ ions was first shown in an earlier work by our group (Pandey et al., 2020). The works for RbBr: $\mathrm{Ca}^{2+}$ system (Sastry \& Sapru, 1980) also report the total elimination of a sharp peak at $466 \mathrm{~K}$ upon $\mathrm{Ca}^{2+}$ doping.

\section{CONCLUSION}

As proposed in Sec. 2, the concept of cation vacancy-anion vacancy pairs seems to meet the requirement of trap centers in alkali halide crystals. Thus the suitability of bi-vacancy as a trap for electrons in $\mathrm{KCl}$ to furnish TL emission is established. The earlier spherical well model, (Herman and Barnet, 1982) however, needed to be modified marginally to fit the potential well for the electron trap to explain the experimental trap depths. Also, a recent proposition (Pandey et al., 2020) suggests that the traps become shallower by the I-V field and draws support from the bi-vacancy model for the traps in $\mathrm{KCl}$. Finally, this work opens up a new era to search for traps suitable for TL emission in $\mathrm{KCl}$.

\section{REFERENCES}

Ausin, V. \& Rivas, J. A. (1972). Thermoluminescence and annealing of F-centres in $\mathrm{KCl}$ gamma irradiated at room temperature. Journal of Physics C: Solid State Physics, 5(1), 82-96.

Bos, A. J. J. (2006). Theory of thermoluminescence. Radiation measurements, 41, S45-S56. 
Benia, H. M., Myrach, P., Gonchar, A., Risse, T., Nilius, N., \& Freund, H. J. (2010). Electron trapping in misfit dislocations of $\mathrm{MgO}$ thin films. Physical Review B, 81(24), 241415.

Bushnell, J. C. (1964). Electron-Nuclear Double Resonance Study of Z (1)Centers in Potassium-Chloride (Doctoral dissertation, University of Illinois at Urbana-Champaign).

Chen, R. (1969). On the calculation of activation energies and frequency factors from glow curves. Journal of Applied Physics, 40(2), 570-585.

Davydov, A. S. (1965). Quantum Mechanics, Pergamon, London.

Deshmukh, B. T., \& Moharil, S. V. (1985). Thermoluminescence of alkali halides doped with alkaline earth impurities. Bulletin of Materials Science, 7(5), 427-457.

Furetta, C. (2003). Handbook of Thermoluminescence. World Scientific, Singapore.

Gourary, B. S., \& Adrian, F. J. (1957). Approximate wave functions for the F center, and their application to the electron spin resonance problem. Physical Review, 105(4), 1180

Herman, Z. S., \& Barnett, G. (1982). The Electronic Structure of F-Centres in Alkali Halide Crystals. Revista Brasileira de Fisica, 12(1), 73-91.

Jain, S. C., \& Mehendru, P. C. (1965). Evidence of Different Types of $F$ Centers from Thermoluminescence Studies in X-Irradiated Alkali Halides. I. Highly Pure $\mathrm{KCl}$ Crystals. Physical Review, 140(3A), A957.
Laad, M. (2009). The Study of Solution Growth of Potassium Bromide Crystal Under Varying Growth Conditions and its Thermoluminescent Properties. International Journal of Mechanics and Solids, 4(1), 63-70.

Pandey, M., Mishra, A. K., Pant, N. \& Pandey, S. D. (2017). Thermoluminescence of undoped and $\mathrm{Mn}^{2+}$ doped $\mathrm{KCl}$. Acta Ciencia India, 43P, 255-260.

Pandey, S., Shripal, Pandey, S. D., Pant, N. \& Kumar, R. (2020). Structure of trap configurations for TL emission in doped $\mathrm{KCL}$ - communicated.

Randall, J. T., \& Wilkins, M. H. F. (1945). Phosphorescence and electron traps-I. The study of trap distributions. Proceedings of the Royal Society of London. Series A. Mathematical and Physical Sciences, 184(999), 365-389.

Ranita, U., Sharma, B. A. \& Singh, S. N. (2006). Application of computerised glow curve deconvolution to determine the spectroscopy of traps in $\mathrm{KCl}$. Indian Journal of Pure and Applied Physics, 44, 308-311.

Sastry, S. B. S., \& Sapru, S. (1980). Thermoluminescence in single crystals of $\mathrm{RbBr}: \mathrm{OH}^{-}$and $\mathrm{RbBr}$ : $\mathrm{Ca}^{2+}$. Pramana, 15(3), 271-278.

Srivastava, J. P. (2008). Solid State Physics. Prentice Hall, New Delhi, India.

Tharmalingam, K., \& Lidiard, A. B. (1961). Mobility of vacancy pairs in ionic crystals. Philosophical Magazine, 6(69), 1157-1162. 
Wosiński, T. (1989). Evidence for the GaAs crystals. Journal of applied electron traps at dislocations in physics, 65(4), 1566-1570.

Table 1. Estimated trap depths (eV) of TL Peaks in different $\mathrm{KCl} / \mathrm{KBr}$ samples

\begin{tabular}{|c|c|c|c|c|}
\hline Sr. No. & Host & Status & $\begin{array}{c}\text { Peak } \\
\text { Temperature } \\
\left({ }^{0} \mathbf{K}\right)\end{array}$ & Trap depth(eV) \\
\hline 1. & $\mathrm{KCl}$ & Negligible I-V pairs & 413 & $\begin{array}{c}0.90 \\
\text { [Pandey et al., 2017] }\end{array}$ \\
\hline 2. & $\mathrm{KCl}$ & $\begin{array}{l}\text { With I-V pairs of stray } \\
\text { impurities } \mathrm{Ca}^{2+}, \mathrm{Ba}^{2+}, \mathrm{Pb}^{2+}\end{array}$ & 361 & $\begin{array}{c}0.44 \\
\text { [Pandey et al., 2017] }\end{array}$ \\
\hline 3. & $\mathrm{KCl}$ & $\begin{array}{l}\text { With I-V pairs of } \\
\text { manganese }\end{array}$ & 339 & $\begin{array}{c}0.48 \\
\text { [Pandey et al., 2017] }\end{array}$ \\
\hline 4. & $\mathrm{KCl}$ & With first type of F-centers & 408 & $\begin{array}{c}1.05 \\
\text { [Jain \& Mahendru, 1965] }\end{array}$ \\
\hline 5. & $\mathrm{KCl}$ & $\begin{array}{l}\text { With second type of } \\
\text { F-centers }\end{array}$ & 463 & $\begin{array}{c}1.15 \\
\text { [Jain \& Mahendru, 1965] }\end{array}$ \\
\hline 6. & $\mathrm{KBr}$ & Undoped crystal & 328 & $\begin{array}{c}0.93 \\
\text { Laad } 200\end{array}$ \\
\hline
\end{tabular}

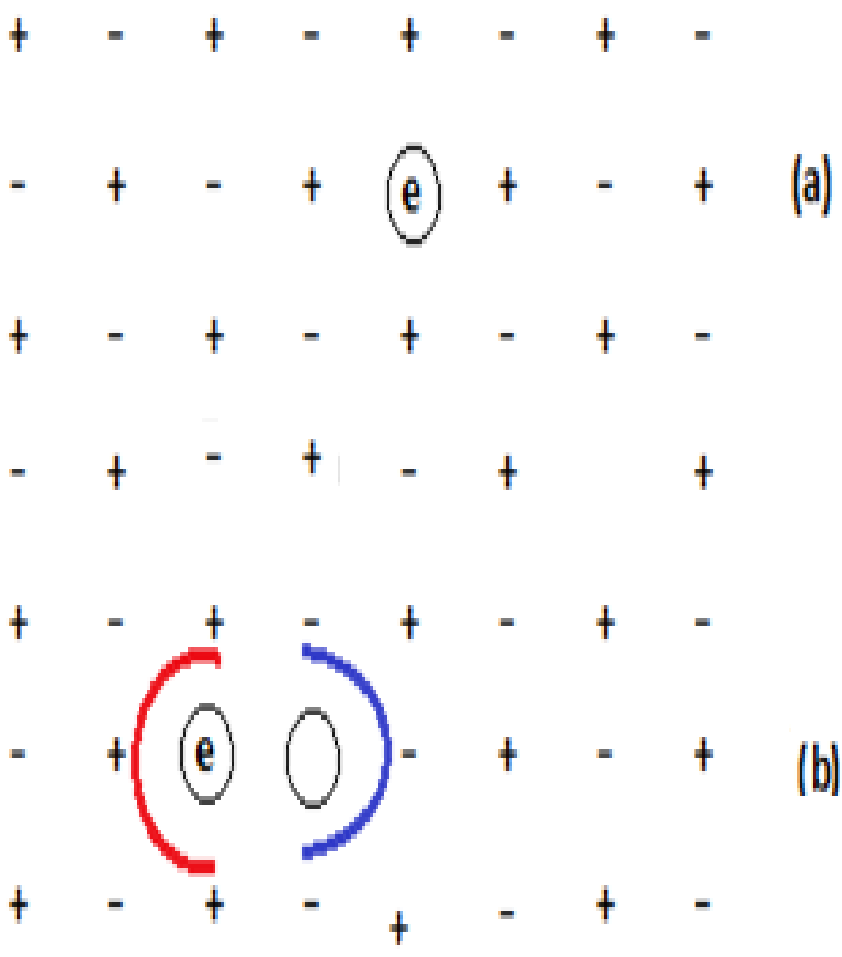




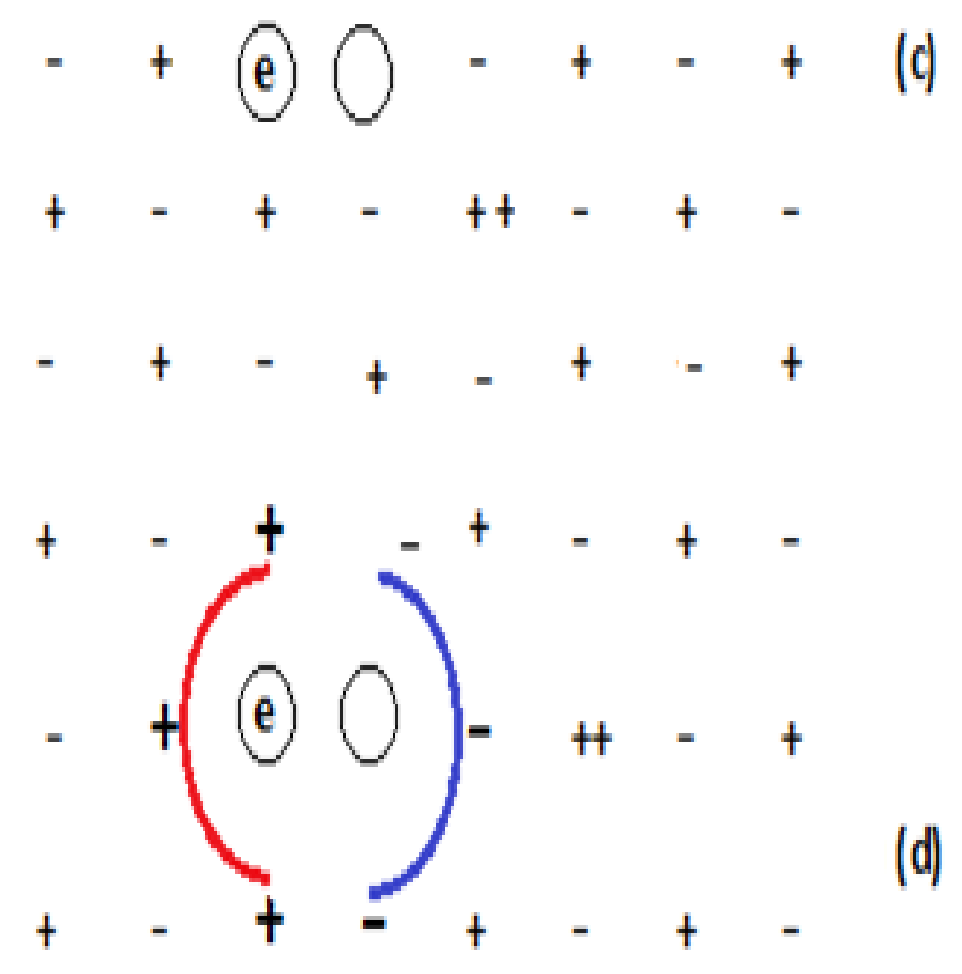

Figure 1. Arrangements of ions in $\mathrm{KCl}$ lattice. Positive alkali ions are shown as "+" and negative halide ions as "_" Possible defects are indicated by markers (a), (b), (c) and (d) viz. (a) Unique F-center, (b) Vacancy - Vacancy pair, (c) F-center perturbed by I-V crystal field in $\mathrm{nn}$ arrangement and (d) F-center perturbed by I-V crystal field in nnn arrangement $/ \mathrm{Z}_{1^{-}}$ center

(a)

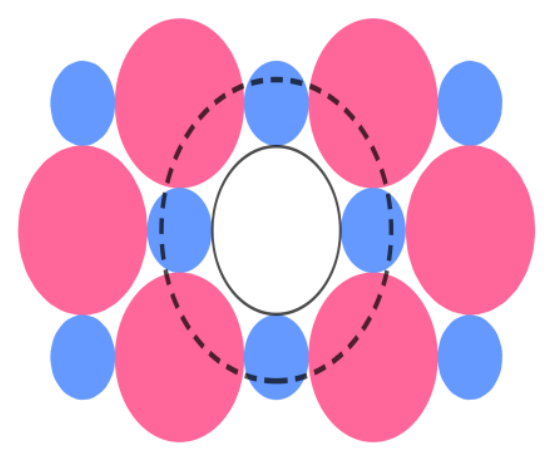

(b)

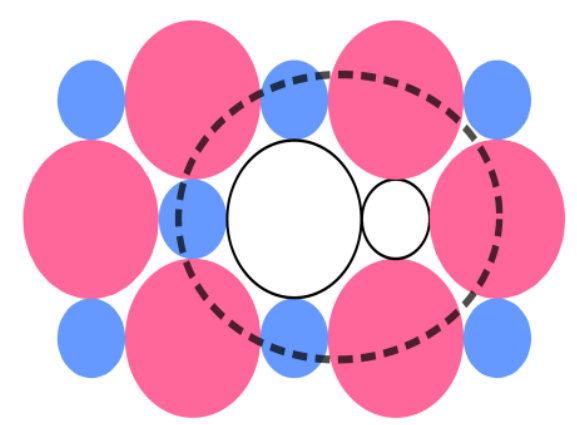

Figure 2. (a) Representation (not to scale) of spherical well by dotted contour, (b) approximaterepresentation (not to scale) of distorted spherical well by dotted contour. In bothcases, the bigger spheres represent $\mathrm{Cl}^{-}$ions and the smaller spheres represent $\mathrm{K}^{+}$ions 